\title{
Surface Path Lines in Plane Stokes Flow Driven by Capillarity
}

\author{
Robert W. Hopper
}

May 3, 1993

This is an informal report intended primarily for internal or limited external distribution. The opinions and conclusionestated are thoce of the author and may or may not be those of the Laboratory.

Work performed under the auspices of the U.S. Department of Energy by the Lawrence Livermore National Laboratary under Contract W-7405-Eng-48. 


\section{DISCLAIMER}

This document was prepared as an account of work sponsored by an agency of the United States Government. Neither the United States Government nor the University of California nor any of their employees, makes any warranty, express or implied, or assumes any legal liability or responsibility for the accuracy, completeness, or usefulness of an information, apparatus, product, or process disclosed, or represents that its use would not infringe privately owned rights. Reference herein to any specific commercial products, process, or service by trade name, trademark, manufacturer, of otherwise, does not necessarily constitute or imply its endorsement, recommendation, or favoring by the United States Government or the University of California. The views and opinions of authors expressed herein do not necessarily state or reflect those of the United States Government or the University of California, and shall not be used for advertising or product endorsement purposes.

This report thas been reproduced directly from the best available copy.

A vailable to DOE and DOE contractors from the Orfice of Scientific and Technical Information P.O. Box 62, Oak Ridge, TN 37831

Prices available from (615) 576-8401, FTS 626-8401

A vallable to the public from the

National Technical Information Service

US. Department of Commerce 5285 Port Royal Rd,

Springfield, VA 22161 


\title{
SURFACE PATH LINES IN \\ PLANE STOKES FLOW DRIVEN BY CAPILLARITY
}

\author{
Robert W. Hopper \\ Chemistry and Materials Science Department \\ Lawrence Livermore National Laboratory \\ Livermore, California 94550
}

\begin{abstract}
Consider the free creeping viscous plane flow in a region, bounded by a simple smooth closed curve and driven solely by surface tensio.1. The shape evolution may in principle, and often in practice, be described by a time-dependent mapping $z=\Omega(\zeta, t)$ of the unit circle, conformal on $|\zeta| \leq 1$. It is shown that the path lines of fluid elements at the surface are determined by a first-order ordinary differential equation involving $\Omega(\zeta, t)$, together with an initial condition. Typically, this must be integrated numerically. Velocities are not needed. The analogous theory for maps from the half-plane $\operatorname{Im} \zeta \geq 0$ is presented. Surface path lines of a collapsing elliptic hole, in two reference frames, are calculated.
\end{abstract}

\section{Introduction and Background}

A path line is the trajectory of a fluid material element. See Truesdell \& Toupin. ${ }^{1}$ Except for steady flows, path lines are not the same as streamlines, which are defined as being everywhere parallel to the instantaneous velocity. Path lines are useful in describing and visualizing a flow, and in determining the history of a fluid element. Path lines are intrinsic in a Lagrangian description; their construction from an Eulerian description entails a numerical integration over the velocity field. This report addresses the path lines of fluid elements at the surface (the "surface path lines") in a special type of moving freeboundary flow: namely, the slow incompressible plane flow of a viscous liquid in a region, hounded by a simple closed curve and driven solely by the surface tension on it. (In the continuum viewpoint, an element at the surface changes shape with time but remains at the moving surface.)

The shape evolution is taken to be described by a time-dependent mapping $z=\Omega(\zeta, t)$ of the unit circle, conformal on $|\zeta| \leq 1$. The path line theory is based on a general theory, developed by the author ${ }^{2-3}$ and axtended by Richardson, ${ }^{4}$ for determining $\Omega(\zeta, t)$ for the above type of flow. Only a small portion of that theory is needed here. When the mapping function $\Omega$ is known, by whatever means, internal fields (velocity, pressure, etc.) can be determined by explicit procedures, but this is usually very laborious. 
One is often content with the shape evolution, possibly supplemented with some surface kinernatics or dynamics, which are easier to calculate. The surface path lines prove surprisingly simple: they are determined by a first-order ordinary differential equation involving $\Omega(\zeta, t)$, together with an initial condition. 'The surface path lines of a collapsing elliptic hole are calculated in Section III as an illustration.

The general theory regards the region as the cross-section of an infinitely long isothermal general cylinder of Newtonian viscous liquid. The basic analyris may be found in Ref. 2, where the assumptions affecting the applicability of the theory to the flow of real liquids are discussed. Solved cases, detailed results and additional references are given in Refs. 2-9. Briefly, the main assumptions are that the flow is planar, that effects of gravity and of inertia are negligible, and that the liquid properties are independent of position and time. Mathematically, it suffices that the curvature of the boundary be a differentiable function of distance along it. A normalization is carried out using a characteristic length of the problem, $L_{0}$. Then the dimensionless position is $z=z_{d} / L_{0}$ and the dimensionless time is $t=\gamma t_{d} / \mu L_{0}$, where $\gamma$ is the surface tension, $\mu$ is the viscosity, and a subscript $d$ denotes the dimensional quantity. The flow is then determined by the dimensionless Stokes equation $\left(\nabla^{2} \mathbf{u}=\nabla p\right)$, the continuity equation $(\nabla \cdot \mathbf{u}=0)$, and the boundary condition that the dimensionless surface traction is normal to the boundary and, in the outward direction, equals the negative of the curvature. Because the theory omits inertial and gravitational effects, rigid-body motions are arbitrary in that the stresses and shape evolution are unaffected. The velocity and rotation at some chosen point may therefore be specified as convenient. Path lines, being dependent on the reference frame, are affected by the choice. Setting the linear and angular momentum of the body to zero usually feels most natural. Understanding the flow can, however, be enhanced by other choices. See Section III, and Ref. 1.

The description of the shape evolution by $z=\Omega(\zeta, t),|\zeta| \leq 1$, is always possible. It is usually convenient to choose the zero of time as a singular case (e.g., the initial contact for coalescing cylinders); the solutions are then valid for $t>0$. The kinematical requirement is that the surface velocity be such that a fluid element at the boundary point $z=\Omega\left(e^{i \vartheta}, t\right)$ moves to $z+d z=\Omega\left[e^{i(\vartheta+\alpha \vartheta)}, t+d t\right]$ in the time increment $d t$. The change $d \vartheta$ depends on $\vartheta$ and $t$ and is expressed $d \vartheta=\dot{\Theta}(\vartheta, t) d t$. This defines $\dot{\Theta}$. (Obtaining the path line will involve a certain integration of $\dot{\Theta}$ over $t$. The overdot in $\dot{\Theta}$ is merely descriptive, however: as defined, there is no function $\Theta$ whose time-derivative is $\dot{\theta}$.) The surface tractions are implicit in the map. One is led to define a function $F(\zeta, t)$, analytic 
on $|\zeta| \leq 1$, whose boundary values provide $\dot{\Theta}$. The result is

$$
\begin{aligned}
\dot{\Theta}(\vartheta, t) & =i \frac{1}{2 \pi i} \oint \frac{1}{2\left|\Omega^{\prime}(\zeta, t)\right|} \frac{\zeta+e^{i \vartheta}}{\zeta\left(\zeta-e^{i \vartheta}\right)} d \zeta \\
& =\frac{1}{4 \pi} \int_{-\pi}^{\pi} \frac{1}{\left|\Omega^{\prime}\left(e^{i \varphi}, t\right)\right|} \frac{\sin (\varphi-\vartheta)}{1-\cos (\varphi-\vartheta)} d \varphi .
\end{aligned}
$$

Here, the primes denote the complex derivative with respect to $\zeta$, the contour in $(1 a)$ is the unit circle $|\zeta|=1$, and the Cauchy principal value is implied. The theory provides a condition, arising from the dynamics and involving $F(\zeta, t)$, that determines the time evolution of $\Omega(\zeta, t)$. In practice, a suitable parametric form $\Omega(\zeta, t)=\Omega\left[\zeta ; a_{1}(t), a_{2}(t), \ldots\right]$ must be conjectured. Generally, the shape-evolution condition provides functional relations between the parameters, and the time is a quadrature over one of them.

\section{Surface Path Lines}

The path lines are obtained by, essentially, integrating $\dot{\Theta}$ over $t$ in a sort of Lagrangian description. Some care in the definitions is needed. Consider then a volume element of fluid which at time $t_{i}$ is located at the surface position $\Omega\left(e^{i \vartheta}, t_{i}\right)$. This element is now labelled $\vartheta$ for all time. Define the function $\Theta_{P L}^{t}\left(t ; \vartheta, t_{i}\right)$ such that at time $t$ the element labelled $\vartheta$ has moved to $\Omega\left\{\exp \left[i \Theta_{P L}^{t}\left(t ; \vartheta, t_{i}\right)\right], t\right\}$. The function $\Theta_{P L}^{t}$ depends on $\vartheta$ and $t_{i}$ only parametrically, these being fixed for a given path line: $t_{i}$ is an arbitrarily chosen initial time, and the particular path line is selected by the choice of $\vartheta$. "Initial" is a slight misnomer, for the definition of $\Theta_{P L}^{t}$ applies to all $t>0$, including times prior to $t_{i}$. The mathematical initial condition is, however, stated for $t=t_{i}$ : Clearly, $\Theta_{P L}^{t}\left(t_{i} ; \vartheta, t_{i}\right)=\vartheta$. It proves useful to express the quantity $\Theta_{P L}$ variously, as functions of different independent variables; the superscript distinguishes between these. All this is just a convenient way of describing the path line. (The elaborate notation will be simplified when no confusion results.)

Now, the element that at time $t$ is at $\Omega\left\{\exp \left[i \Theta_{P L}^{t}\left(t ; \vartheta, t_{i}\right)\right], t\right\}$ moves, according to the definition of $\dot{\Theta}$, to $\left.\Omega\left(\exp \left\langle i\left\{\Theta_{P I}^{t}\left(t ; \vartheta, t_{i}\right)+\dot{\Theta}\left[\Theta_{P L}^{t}\left(t ; \vartheta, t_{i}\right), t\right)\right] d t\right\}\right\rangle, t+d t\right)$ during the time interval $(t, t+d t)$. This means that the time derivative of $\Theta_{P L}^{t}$ is $\dot{\Theta}\left(\Theta_{P L}^{t}, t\right)$. Note that $\dot{\Theta}$ does not depend explicitly on $\vartheta$ or $t_{i}$ but only implicitly through $\Theta_{P L}^{t}$. The time-evolution of $\Theta_{P L}^{t}$ - of the surface path line - is thus governed by the nonlinear ordinary differential equation

$$
d \Theta_{P L}^{t}(t) / d t=\dot{\Theta}\left[\Theta_{P L}^{t}(t), t\right]
$$


together with the initial condition

$$
\Theta_{P L}^{\ell}\left(t_{i}\right)=\vartheta
$$

Consider now the choice of $t_{i}$. For practical computation, it doesn't really matter: one may simply choose any $t_{i}>0$ and integrate both forward and backwards in time. It would be more pleasing, however, to choose $t_{i}=0$. Though this seems natural, it is problematical because, usually, the map $\Omega(\zeta, t)$ is by choice singular $2^{t} t=0$. One reflection of this singular behavior is that points equidistant on $|\zeta|=1$ (i.e., at equal intervals of $\vartheta$ ) are "crowded" into cusp-like regions in $z$-space as $t \rightarrow 0$. (Cusps are the only singularities of the regions for which the theory has thus far succeeded.) Correspondingly, $\dot{\Theta}$ blows up as $t \rightarrow 0$. Handling the problem ad hoc with small-t expansions would seem perverse. The obvious alternative is to choose $t_{i}=\infty$; that is, to label the path lines with their final values of $\vartheta$. Direct integration backwards from $t=\infty$ would be clumsy, however, because now $\dot{\Theta} \rightarrow 0$. A transformation of the time is indicated. This may in general require special treatment, but it usually suffices simply to regard the motion along the path line as a function of the time-controlling parameter, recovering $t$ from the appropriate formula.

Suppose that the time is specified by a parameter $v$ which decreases from 1 to 0 over time. Then the transformation takes the form

$$
\begin{gathered}
\Theta_{P L}^{v}(v)=\Theta_{P L}^{t}[t(v)], \\
d \Theta_{P L}^{v}(v) / d v=\dot{\Theta}\left[\Theta_{P L}^{v}(v), t(v)\right][d t(v) / d v], \\
\Theta_{P L}^{v}(0)=v .
\end{gathered}
$$

The initial-value problem ( $3 b-c)$ has been solved numerically for the case of coalescing equal cylinders. ${ }^{9}$ No difficulties were encountered.

The preceding treatment was for maps from $|\zeta| \leq 1$. This is the natural reference domain when the liquid occupies a finite simply-connected region, an infinite region with a hole, or half-space with a periodic boundary. When the liquid occupies a half-space with an isolated disturbance, a map from $\operatorname{Im} \zeta \geq 0$ is more suitable. 'The analysis ${ }^{3}$ is very similar, and the path lines can be treated briefly. Let $\xi=\operatorname{Re} \zeta$. A fluid element which at 1 ime $t$ is at $\Omega(\xi, t)$ moves to $\Omega[\xi+\dot{\Xi}(\xi, t) d t, t+d t]$ during $d t$. This defines $\dot{\Xi}$. Then

$$
\dot{\Xi}\left(\xi_{0}, t\right)=\frac{1}{2 \pi} \int_{-\infty}^{\infty} \frac{1}{\left|\Omega^{\prime}(\xi, t)\right|} \frac{1}{\xi-\xi_{0}} d \xi
$$


Label the fluid element that at time $t_{i}$ is located at $\Omega\left(\xi, t_{i}\right)$ by $\xi$. Define $\Xi_{P L}^{t}\left(t ; \xi, t_{i}\right)$ by the condition that at time $t$ this element has moved to $\Omega\left[\Xi_{P L}^{t}\left(t ; \xi, t_{i}\right), t\right]$. Then reasoning analogous to the preceding leads to

$$
d \Xi_{P L}^{t}(t) / d t=\dot{\Xi}\left[\Xi_{P L}^{t}(t), t\right], \quad \Xi_{P L}^{t}\left(t_{i}\right)=\xi
$$

Again choosing $t_{i}=\infty$, transform the problem to one using the time-controlling parameter, say $h$, as the independent parameter, defining a corresponding path line function $\Xi_{P L}^{h}(h)$. Supposing that $h$ also decreases to zero over time,

$$
\begin{gathered}
\Xi_{P L}^{h}(h)=\Xi_{P L}^{t}[t(h)], \\
d \Xi_{P L}^{h}(h) / d h=\dot{\Xi}\left[\Xi_{P L}^{h}(h), t(h)\right][d t(h) / d h], \\
\Xi_{P L}^{h}(0)=\xi .
\end{gathered}
$$

The path lines for the coalescence of a cylinder with half-space, computed from these equations, are included in Ref. 9.

The analysis is not essentially affected by the choice of the rigid-body motion. They are simply represented as a translation and rotation (which needn't be uniform in time) of the $z$-space coordinates, and incorporated into $\Omega(\zeta, t)$. The various path line functions $\left(\Theta_{P L}^{t}\right.$, etc.) do not change.

\section{Elliptic Hole}

The flow of an infinite region containing an elliptic hole $e^{\delta}$ provides a simple, though in some ways atypical, example. The hole remains elliptical, with the axes shortening at constant speeds; so the eccentricity doesn't change, and the hole closes in a finite time. Geometrically similar points on successive ellipses are images of the same $\zeta$-space point: they have the same value of $\vartheta$. This does not, however, imply that the material elements at these points are the same; and, in fact, they are not.

The analysis in Ref. 3 may be summarized, in a slightly cleaner form, as follows: Let the ratio of the axes be $m,(0<m \leq 1)$. Then the eccentricity of the ellipse is $k=\left(1-m^{2}\right)^{1 / 2}$. Let the dimensional time and semi-major axis length be $t_{d}$ and $L_{d}\left(t_{d}\right)$ respectively. Since there is no "natural" zero of time, select it arbitrarily; and choose as the characteristic dimension $L_{0}$ the corresponding length of the semi-major axis: $L_{0}=L_{d}(0)$. The dimensionless length of the semi-major axis is then $L=L_{d} / L_{0}$, and that of the semi-minor axis is $m L . L$ is used as the time-controlling parameter; $L(0)=1$. Using Landen's transformation, the earlier forms may be expressed 


$$
\begin{gathered}
\Omega(\zeta, t)=[L(t) / 2]\left[(1+m) \zeta^{-1}+(1-m) \zeta\right],|\zeta| \leq 1, \\
L(t)=1-K(k) t / \pi .
\end{gathered}
$$

The hole vanishes at $t=\pi / K(k)$, so the results apply to $t$ on $[-\infty, \pi / K(k)]$. The centroid is stationary and there is no rigid rotation (zero velocity at infinity). By symmetry, the treatment to confined to $0 \leq \vartheta \leq \pi / 2$. (Increasing $\vartheta$ corresponds to the clockwise direction around the ellipse.)

From (7),

$$
\left|\Omega^{\prime}\left(e^{i \vartheta}, t\right)\right|=4 \pi L(t)\left(1-k^{2} \cos ^{2} \vartheta\right)^{1 / 2}
$$

Then direct application of (1b) gives, with the use of eq. 13.8(21) in Ref. 10,

$$
\dot{\Theta}(\vartheta, t)=\frac{1}{\pi L(t)}\left(1-k^{2} \cos ^{2} \vartheta\right)^{-1 / 2}\left[\mathbf{K}(k) E\left(\frac{\pi}{2}-\vartheta, k\right)-\mathbf{E}(k) F\left(\frac{\pi}{2}-\vartheta, k\right)\right] .
$$

Here $F(\phi, k), E(\phi, k), \mathbf{K}(k)$ and $\mathbf{E}(k)$ are Legendre normal elliptic integrals. With eqs. 3 and 8 , the path line equation becomes

$$
\frac{d \Theta_{P L}^{L}(L)}{d L}=\frac{\mathbf{K}(k) E\left(\frac{\pi}{2}-\vartheta, k\right)-\mathbf{E}(k) F\left(\frac{\pi}{2}-\vartheta, k\right)}{L K(k) \sqrt{\left.1-k^{2} \cos ^{2} \Theta_{P L}^{L}\right)}} .
$$

With the initial condition $\Theta_{P L}^{L}(L ; \vartheta, 1)=\vartheta$, this integrates to give $\Theta_{P L}^{L}$ as an implicit function of $L$. Thus, for a given path line $\vartheta$,

$$
L=\exp \left[K(k) \int_{\pi / 2-\Theta_{P L}^{L}}^{\pi / 2-\vartheta} \frac{\sqrt{1-k^{2} \sin ^{2} \varphi}}{\mathbf{K}(k) E(\varphi, k)-\mathbf{E}(k) F(\varphi, k)} d \varphi\right] .
$$

Since $\dot{\Theta}(0, t)=\dot{\Theta}(\pi / 2, t)=0$, eq. 12 holds for $0<\vartheta<\pi / 2,0<\Theta_{P L}^{L}<\pi / 2$; and $\Theta_{P L}^{L}(L ; 0,1)=0$, $\Theta_{P L}^{L}(L ; \pi / 2,1)=\pi / 2$.

Repetitive computation of this integral for different path lines $\vartheta$ would be inefficient, so define

$$
H_{L}(\theta, k)=\int_{\pi / 4}^{\pi / 2-\theta} \frac{\sqrt{1-k^{2} \sin ^{2} \varphi}}{\mathbf{K}(k) E(\varphi, k)-\mathbf{E}(k) F(\varphi, k)} d \varphi, \quad 0<\theta<\pi / 2 .
$$

Then

$$
L=\exp \left\{K(k)\left[H_{L}(\vartheta, k)-H_{L}\left(\Theta_{P L}^{L}, k\right)\right]\right\}
$$


Figure 1 shows $\Theta_{P L}$ as a function of $L$ and of $t$, for several values of $m$ and $\vartheta$, while Fig. 2 shows the surface path lines for $m=1 / 2$. By replacing $\Omega(\zeta, t)$ with $\Omega(\zeta, t)+L(t)$, a rigid translation is imposed which fixes the left-hand apex of the ellipse. Using eqs. 13-14 unchanged, the path lines in Fig. 3 are obtained. For aesthetics, the images in Figs. 2 and 3 have been reflected across the Re- $z$ axis.

When the eccentricity $k=0$, and the hole is circular, the path lines are obviously radii: $\Theta_{P L}^{L}(L ; \vartheta, 1)=\vartheta, L>0$. The case $k=1$ is the infinite region exterior to a parabola. It has been shown ${ }^{6}$ that, in the absence of extraneous conditions at infinity, the parabola translates at a constant speed without change of shape. In a reference frame whose origin coincides with the apex of the parabola, the interior path lines are just the streamlines, and there is but a single surface path line - the parabola itself. This result can be seen in the fixed-apex case (Fig. 3) by rescaling and allowing $k \rightarrow 1$.

\section{Discussion}

In general, for any plane or axisymmetric free-boundary flow in a simplyconnected region, it is always possible to describe the shape by a time-dependent conformal map. Given the map, the path lines can always be described by some $\Theta_{P L}^{t}\left(t ; \vartheta, t_{i}\right)$. The utility of the latter description (ignoring that finding the map is in general formidable) depends on whether $\Theta_{P L}^{t}$ is governed by a tractable law. For example, Antanovskii ${ }^{11}$ has developed another theoretical approach for plane Stokes flows, employing the bianalytic stress-stream function, time-dependent conformal maps, and the boundary integral method. The present theory would be applicable to maps obtained by his methods for flows driven solely by a constant surface tension. To the author's knowledge, however, the cases treated to date are more complicated, and for them $\dot{\Theta}$ is not known.

Time-dependent conformal mapping has proved useful in treating Hele-Shaw flows, and also certain other Lapacian fields. The author has not attempted to extract a $\Theta_{P L}$ from these theories. The use of a time-dependent Schwarz function is another natural way to describe the shape evolution of plane free-boundary flows. Path lines in a Schwarz function description do not seem to have been investigated. The recent overviev' by Howison ${ }^{12}$ provides additional references. 


\section{References}

1. C. Truesdell \& R. Toupin, "The Classical Field Theories" in Encyclopedia of Physics,

S. Flügge, ed., vol. 3, pt. 1 (1960). Sections 70-71.

2. R. W. Hopper, "Plane Stokes flow driven by capillarity on a free surface," J. Fluid Mech. 213, 349-375 (1990). Errata are given in Refs. 3 \& 5.

3. R. W. Hopper, "Plane Stokes flow driven by capillarity on a free surface, Part 2:

Further developments," J. Fluid Mech. 230, 355-364 (1991). Errata are given in Ref. 5.

4. S. Richardson, "Two-dimensional slow viscous flows with time-dependent free boundaries driven by surface tension," Europ. J. App. Math. 3, 193-207 (1992).

5. R. W. Hopper, "Stokes flow of a cylinder and half-space driven by capillarity," J. Fluid Mech. 243, 171-81 (1992)

6. R. W. Hopper, "Capillarity-driven plane Stokes flow exterior to a parabola," Q. J. Mech. appl. Math. (1993). To appear.

7. R. W. Hopper, "Coalescence of two viscous cylinders by capillarity, Part 1: Theory," J. Am. Ceram. Soc. (1993). Submitted.

8. R. W. Hopper,"Coalescence of two viscous cylinders by capillarity, Part 2: Shape evolution," J. Am. Ceram. Soc. (1993). Submitted.

9. R. W. Hopper, "Coalescence of two viscous cylinders by capillarity, Part 3: Flow at the surface," J. Am. Ceram. Soc. (1993). To be submitted.

10. A. Erdélyi, ed., Higher Transcendental Functions, vol. 2. New York: McGraw-Hill Book Co., 1953.

11. L. K. Antanovskii, "Boundary integral equations in quasisteady problems of capillary fluid mechanics, Part 2: Applicaton of the stress-stream function," Meccanica 26, 59-65 (1991).

12. S. D. Howison, "Complex variable methods in Hele-Shaw moving boundary problems," Europ. J. App. Math. 3, 209-224 (1992). 


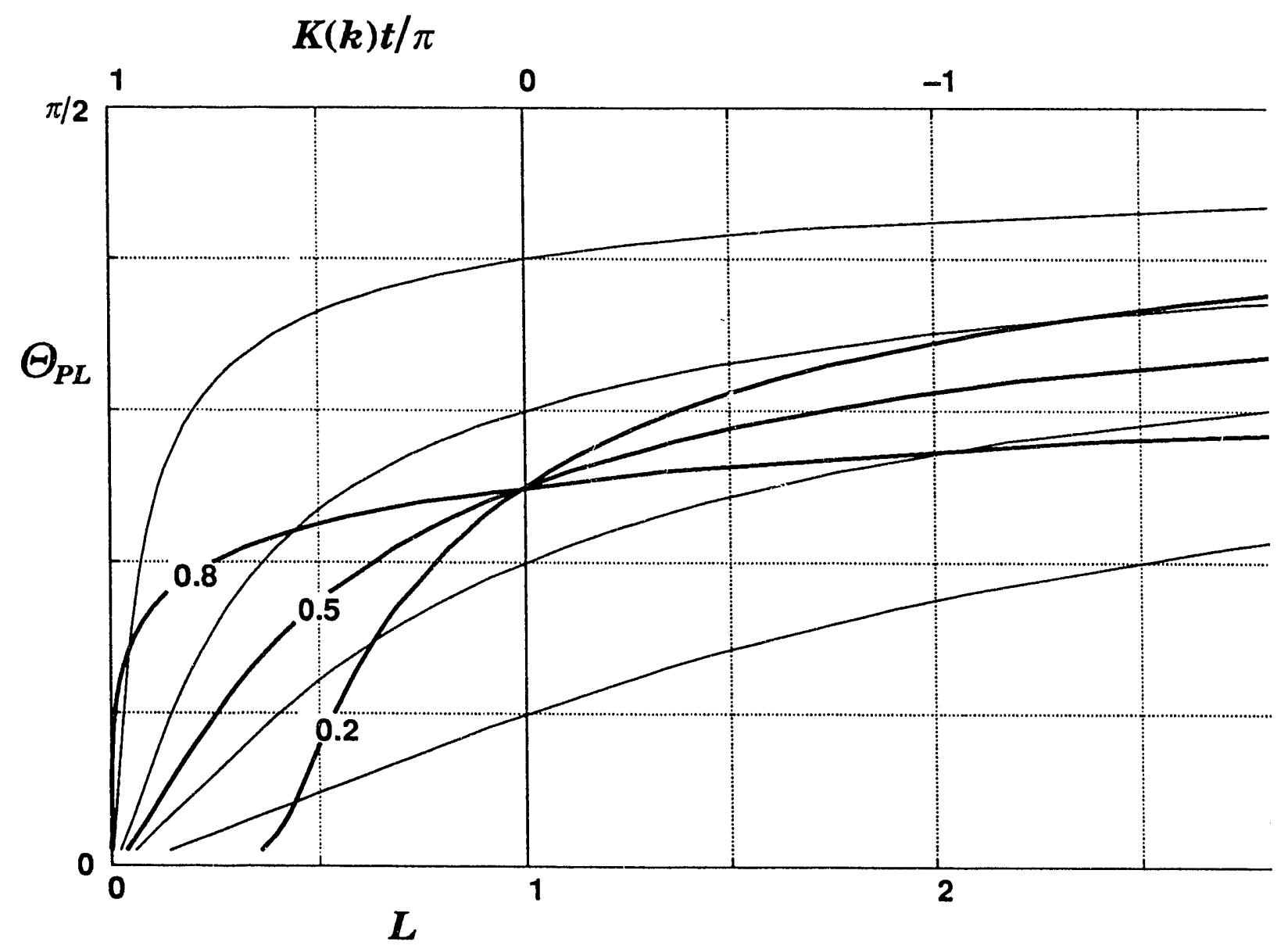

Figure 1. The path line function $\Theta_{P L}$ as a function of $L$ (lower abscissa) and $t$ (upper) for the collapsing elliptic hole. Light curves: $m=0.2, \vartheta=(0.1 \pi, 0.2 \pi, 0.3 \pi \& 0.4 \pi)$. Heavy curves: $\vartheta=0.25 \pi, m=(0.2,0.5 \& 0.8)$. For $m=1, \Theta_{P L}=\vartheta$ independent of $L$. 


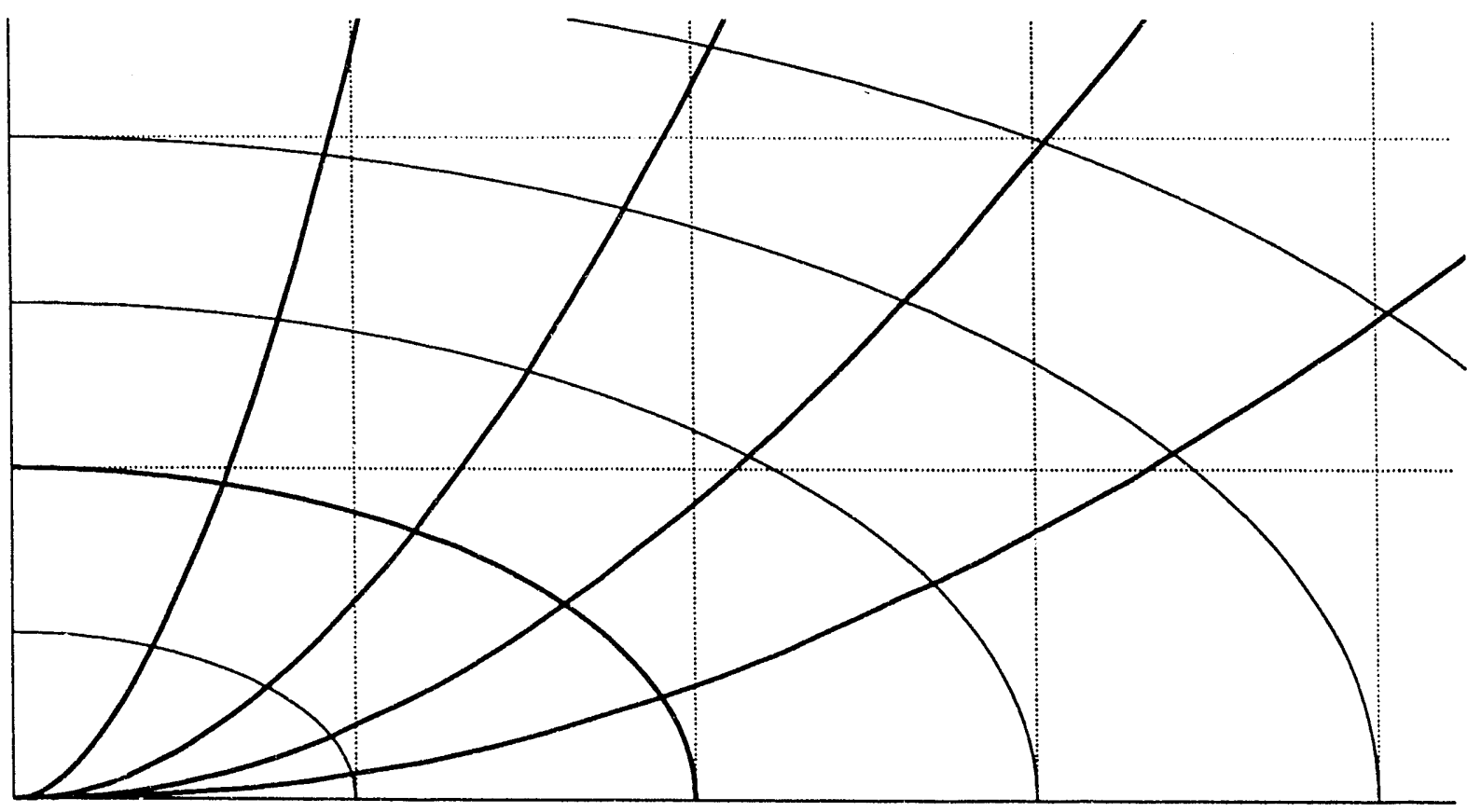

Figure 2. Surface path lines of a collapsing elliptic hole with no translation or rotation, for $m=0.5$. The hole shapes are at equal intervals of time, the heavy shape being that for $t=0(L=1)$. (Grid intervals are 0.5.) The path lines are for $\vartheta=(0.1 \pi, 0.2 \pi, 0.3 \pi \& 0.4 \pi)$, lower right to upper left. Path lines for $\vartheta=(0 \& \pi / 2)$ coincide with the axes. 


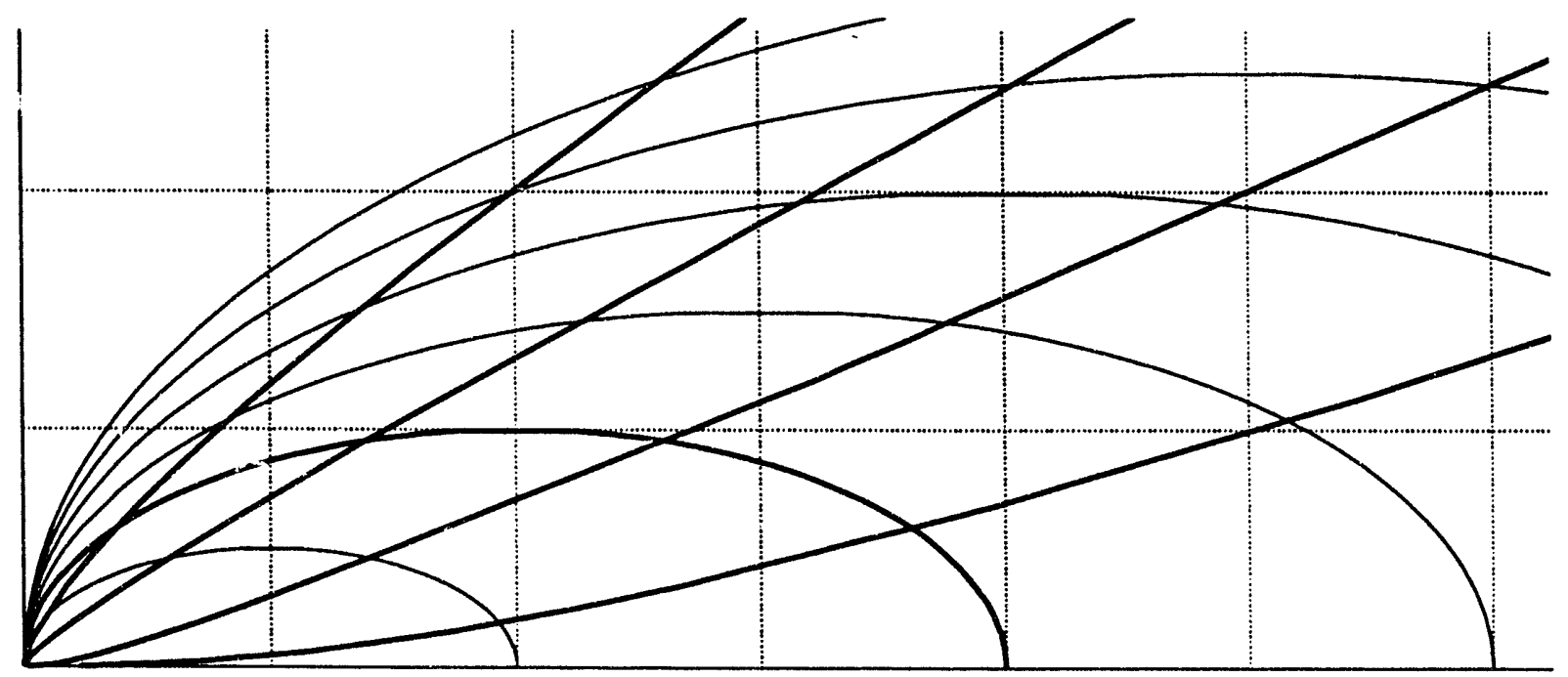

Figure 3. Surface path lines of a collapsing elliptic hole with the left-hand apex fixed, for $m=0.5$. The path lines are for $\vartheta=(0.2 \pi, 0.4 \pi, 0.6 \pi \& 0.8 \pi)$, lower right to upper left. The hole shapes are at equal intervals of time, the heavy shape being that for $t=0$ $(L=1)$. (Grid intervals are 0.5 .) The path line for $\vartheta=0$ coincides with the abscissa; that for $\vartheta=\pi / 2$ is a straight line through the intercepts of the ellipses with their respective minor axes; that and that for $\vartheta=\pi$ has degenerated to a point at the origin. 

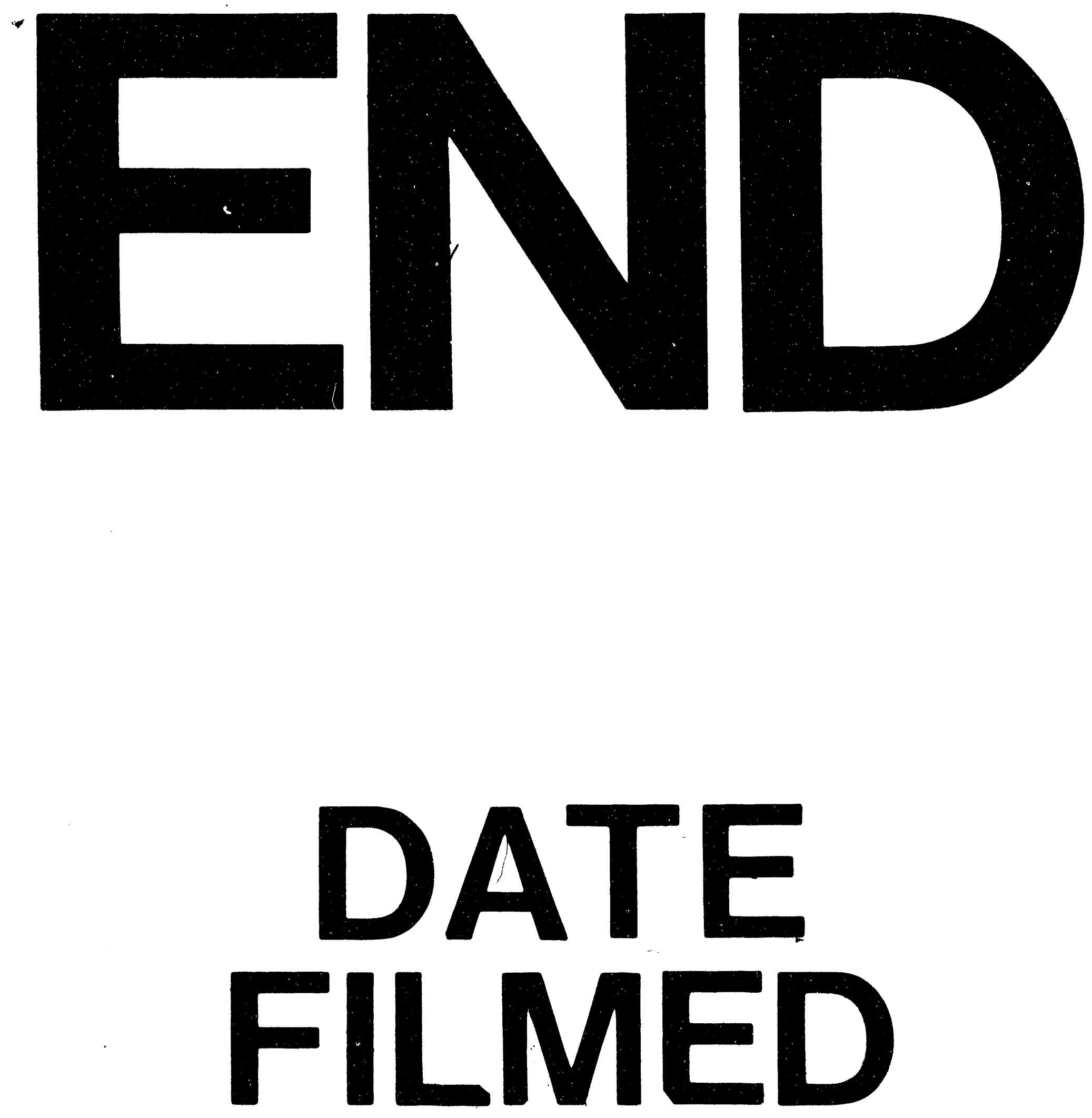

1

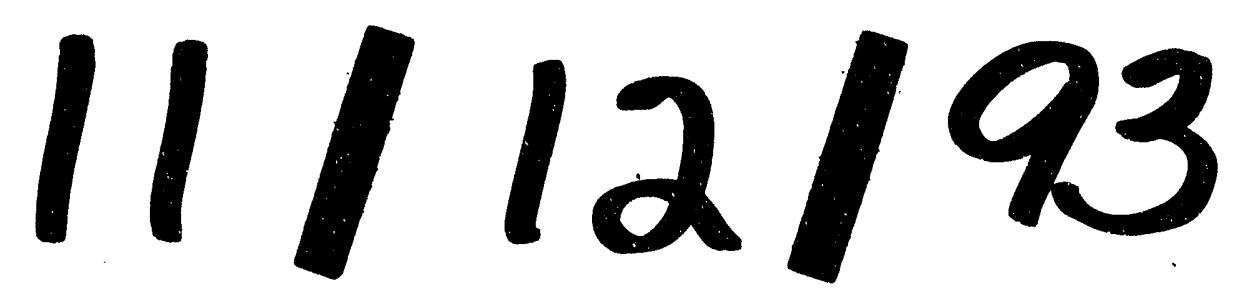


